\title{
Triplet and reversed triplet mechanism CIDEP studied by quenching experiments
}

\author{
Savitsky, A N ; Batchelor, S N ; Paul, H
}

\begin{abstract}
Quenching experiments are shown to provide a convenient tool to check for the presence of triplet mechanism (TM) spin polarisation in time-resolved EPR spectra following laser flash photolysis. The effect of the triplet quenchers, trans-1,3-pentadiene, fumaronitrile, azo-tert-butane and azo-n-butane upon the spectra following laser photolysis of acetone/propan-2-ol and benzophenone/propan-2-ol photosystems show that no TM polarisation is present in the former system but emissive TM is present in the latter. Use of 2,2 -azo-bis[isobutryronitrile] produces an anomalous emissive polarisation upon quenching, which is tentatively attributed to a reversed TM in the triplet sensitised azo-compound
\end{abstract}

DOI: https://doi.org/10.1007/bf03162208

Posted at the Zurich Open Repository and Archive, University of Zurich ZORA URL: https://doi.org/10.5167/uzh-156332

Journal Article

Published Version

Originally published at:

Savitsky, A N; Batchelor, S N; Paul, H (1997). Triplet and reversed triplet mechanism CIDEP studied by quenching experiments. Applied Magnetic Resonance, 13(3-4):285-295.

DOI: https://doi.org/10.1007/bf03162208 


\title{
Triplet and Reversed Triplet Mechanism CIDEP Studied by Quenching Experiments
}

\author{
A. N. Savitsky, S. N. Batchelor, and H. Paul \\ Physikalisch-Chemisches Institut der Universität Zürich, Zürich, Switzerland \\ Received May 14, 1997; revised June 9, 1997
}

Dedicated to Harry Kurreck on the occasion of his 65 th birthday

\begin{abstract}
Quenching experiments are shown to provide a convenient tool to check for the presence of triplet mechanism (TM) spin polarisation in time-resolved EPR spectra following laser flash photolysis. The effect of the triplet quenchers, trans-1,3-pentadiene, fumaronitrile, azo-tert-butane and azo-n-butane upon the spectra following laser photolysis of acetone/propan-2-ol and benzophenone/propan-2-ol photosystems show that no TM polarisation is present in the former system but emissive TM is present in the latter. Use of $2,2^{\prime}$-azo-bis[isobutryronitrile] produces an anomalous emissive polarisation upon quenching, which is tentatively attributed to a reversed TM in the triplet sensitised azo-compound.
\end{abstract}

\section{Introduction}

Quenching experiments are widely used in the unraveling of photochemical reaction mechanisms [1]. In general, a transient intermediate such as a photoexcited triplet is quenched by addition of a suitable compound and the effect of this upon reaction observed. Time-resolved EPR (TREPR) following flash photolysis is a widely used experiment for investigating radical photochemistry, however little work has been done with quenchers [2-4]. This is surprising as often a major problem in TREPR is the identification of the origin of net absorptive (A) contributions in spectra arising from triplet precursors, where quenching experiments should provide key information.

Net A contributions are often assigned to the Triplet Mechanism (TM) where the polarisation is produced in a photoexcited triplet by level selective intersystem crossing from the initially excited singlet. This polarisation may then be transferred to the radicals, if the triplet reacts before spin lattice relaxation $\left({ }^{3} T_{1}\right)$ has destroyed the polarisation. Fast reactions are therefore required, as ${ }^{3} T_{1}$ 's are thought to be a few nanoseconds in solution [4-8]. However, net A contributions in TREPR spectra may also arise simply from the Boltzmann signal of the radicals or transfer of the Boltzmann polarisation of the triplet precursor. 
Distinguishing between the two mechanisms is extremely difficult, as exemplified par excellence by the photoreduction of acetone by propan-2-ol. This system has been intensely studied by the TREPR community for over 20 years [9-11]. The resulting 2-hydroxy-2-propyl radicals are observed in an emission/ absorption (E/A) multiplet overlaid upon a net A contribution. The E/A pattern is accepted to arise from the Radical Pair Mechanism (RPM), however the origin of the net $A$ contribution has been fiercely debated [3, 9-14]. Variation of the triplet lifetime $\left(\tau_{\tau}\right)$ by addition of triplet quenchers should provide a simple qualitative means to distinguish between the two mechanisms. Addition of triplet quenchers will of course diminish both multiplet and net polarisation, as triplet molecules will be partially removed. However, as the quencher also shortens $\tau_{\mathrm{T}}$, the relative contribution of any net polarisation due to the TM should increase because of its dependence on ${ }^{3} T_{1}$. Here, this possibility is investigated using the acetone/propan-2-ol and analogous benzophenone/propan2-ol photosystems with trans-1,3-pentadiene, fumaronitrile, azo-tert-butane, azon-butane and 2,2'azo-bis[isobutryronitrile] (AIBN) as triplet quenchers. The first four quench triplets without formation of radicals, whereas triplet AIBN partially reacts to produce two, 2-cyano-2-propyl radicals $[15,16]$ :

$$
{ }^{3} \mathrm{~K}+\mathrm{AIBN} \longrightarrow \mathrm{K}+{ }^{3} \mathrm{AIBN} \longrightarrow \mathrm{K}+\mathrm{N}_{2}+2\left(\mathrm{CH}_{3}\right)_{2} \mathrm{C} \cdot \mathrm{CN} \text {, }
$$

where ${ }^{3} \mathrm{~K}$ denotes a triplet ketone. To account for the variation in initial radical concentrations with quencher concentration, a method is introduced whereby the net contribution is normalized to the size of the multiplet polarisation. Theoretical calculation shows that in the concentration ranges used, this multiplet polarisation is proportional to the radical concentration to a good approximation.

\section{Experimental}

Time-resolved EPR spectra were obtained using a spectrometer described previously [17]. It consists of an excimer (XeCl, $308 \mathrm{~nm}, 15 \mathrm{~ns}$ pulse width) or NdYAG (355 nm, $6 \mathrm{~ns}$ pulse width) laser and an X-band cw EPR spectrometer (80 ns response time). Solutions were deoxygenated by argon purging and afterwards exposed to the laser light $(10 \mathrm{~Hz}, 5-15 \mathrm{~mJ}$ per pulse on sample surface) whilst flowing through a quartz cell in the TE103 EPR cavity $(1 \mathrm{~mm}$ optical path length). Acetone solutions were excited by the excimer and benzophenone by the Nd-YAG laser, optical densities were $0.2-0.6$ and care was taken that the triplet quenchers absorbed less than $1 \%$ of the light. Signals were acquired using a Le Croy 9310A oscilloscope and transferred to a PC. Solutions were checked by UV absorption spectroscopy before and after irradiation to ensure minimal depletion of the substrates.

Chemicals were obtained from Aldrich and Fluka and used as purchased. 


\section{Theory}

In order to characterise the effect of triplet quenchers on the TM contribution to TREPR spectra, simulation of time profiles were performed. These require a knowledge of the chemistry, rate constants, relaxation times and polarisations. For the acetone and benzophenone/propan-2-ol systems investigated here these parameters are reasonably well known from previous studies. In both cases the ketones rapidly intersystem cross into the triplet state upon excitation, within picoseconds for benzophenone and nanoseconds for acetone [18]. The triplet state then abstracts a proton from propan-2-ol with a rate constant $k_{\mathrm{a}}$ :

$$
{ }^{3} \mathrm{~K}+\left(\mathrm{CH}_{3}\right)_{2} \mathrm{CHOH} \stackrel{k_{\mathrm{a}}}{\longrightarrow} \mathrm{K}^{\cdot} \mathrm{H}+\left(\mathrm{CH}_{3}\right)_{2} \mathrm{C}^{\cdot} \mathrm{OH}
$$

and the resulting radicals react by bimolecular termination, $2 k_{\mathrm{r}}$. Addition of a triplet quencher, $\mathrm{Q}$, quenches the ketone triplet with a rate constant $k_{\mathrm{q}}$ :

$$
{ }^{3} \mathrm{~K}+\mathrm{Q} \stackrel{k_{\mathrm{q}}}{\longrightarrow} \mathrm{K}+{ }^{3} \mathrm{Q} .
$$

The radicals are produced with a net contribution, $M_{z}^{0}$, to the $z$-component of the magnetic moment, arising from the TM and/or Boltzmann polarisation of the triplet. This may be calculated either via modification of formulae given by Atkins and Evans [6] or by integrating the following differential Eqs. (4)-(7). Here $P^{\mathrm{T}}$ is the relaxing triplet polarisation and, in order to simplify the scheme, only the 2-hydroxy-2-propyl radicals are considered leading to $x=1$ for benzophenone and $x=2$ for acetone in Eq. (6):

$$
\begin{aligned}
\frac{\mathrm{d} P^{\mathrm{T}}}{\mathrm{d} t} & =-\frac{P^{\mathrm{T}}-P_{\mathrm{eq}}^{\mathrm{T}}}{{ }^{3} T_{1}}, \\
\frac{\mathrm{d}\left[{ }^{3} \mathrm{~K}\right]}{\mathrm{d} t} & \left.=-k_{\mathrm{a}}\left[{ }^{3} \mathrm{~K}\right]\left[\left(\mathrm{CH}_{3}\right)_{2} \mathrm{CHOH}\right)\right]-k_{\mathrm{q}}\left[{ }^{3} \mathrm{~K}\right][\mathrm{Q}], \\
\frac{\mathrm{d}\left[\left(\mathrm{CH}_{3}\right)_{2} \mathrm{C}^{\cdot} \mathrm{OH}\right]}{\mathrm{d} t} & \left.=x k_{\mathrm{a}}\left[{ }^{3} \mathrm{~K}\right]\left[\left(\mathrm{CH}_{3}\right)_{2} \mathrm{CHOH}\right)\right], \\
\frac{\mathrm{d} M_{z}^{0}}{\mathrm{~d} t} & =P^{\mathrm{T}}(t) \frac{\mathrm{d}\left[\left(\mathrm{CH}_{3}\right)_{2} \mathrm{C}^{\cdot} \mathrm{OH}\right]}{\mathrm{d} t} .
\end{aligned}
$$

To be strictly correct the equation system would require a very short laser pulse and extremely fast intersystem crossing. For the present qualitative treatment it should suffice to give a reasonable estimate of $M_{z}^{0}$ as well as the radical and triplet concentration following a $5 \mathrm{~ns}$ laser flash. $\mathrm{A}{ }^{3} T_{1}$ of $0.5 \mathrm{~ns}$ is assumed 
and the initial triplet concentration $\left[{ }^{3} \mathrm{~K}\right]_{0}$ estimated from the optical density and laser power. The initial polarisation $P_{0}^{\mathrm{T}}$ was taken as a free parameter. Values for $k_{\mathrm{a}}$ and $k_{\mathrm{q}}$ were obtained from literature and for acetone are $k_{\mathrm{a}}=1 \cdot 10^{6} \mathrm{M}^{-1} \mathrm{~s}^{-1}$ [19] and $k_{\mathrm{q}}=46,60,3,6$, and $5 \cdot 10^{8} \mathrm{M}^{-1} \mathrm{~s}^{-1}$ for pentadiene, fumaronitrile, azon-butane, azo-tert-butane and AIBN, respectively [1, 20, 21]. For benzophenone $k_{\mathrm{a}}=1 \cdot 10^{6} \mathrm{M}^{-1} \mathrm{~s}^{-1}$ and $k_{\mathrm{q}}=55$ and $8 \cdot 10^{8} \mathrm{M}^{-1} \mathrm{~s}^{-1}$ for pentadiene and azo-tertbutane, respectively $[19,20]$. The values of $M_{z}^{0},\left[{ }^{3} \mathrm{~K}\right]$ and $\left.\left[\left(\mathrm{CH}_{3}\right)_{2} \mathrm{C}^{\cdot} \mathrm{OH}\right)\right]$ thus obtained are then used as initial conditions for the simulation of the microsecond TREPR time profiles. This is done using a modified Bloch equation approach [22-24]

$$
\begin{aligned}
\frac{\mathrm{d} v}{\mathrm{~d} t}= & -\frac{v}{T_{2}}+\omega_{1} M_{z} \\
\frac{\mathrm{d} M_{z}}{\mathrm{~d} t}= & -\omega_{1} v+\frac{P_{\mathrm{eq}}\left[\left(\mathrm{CH}_{3}\right)_{2} \mathrm{C}^{\circ} \mathrm{OH}\right]-M_{z}}{T_{1}}+2 k_{t}\left[\left(\mathrm{CH}_{3}\right)_{2} \mathrm{C}^{\cdot O H}\right]^{2} P_{\mathrm{F}} P_{\mathrm{eq}} \\
& \left.+k_{\mathrm{a}}{ }^{3} \mathrm{~K}\right]\left[\left(\mathrm{CH}_{3}\right)_{2} \mathrm{CHOH}\right]\left(P_{\mathrm{G}}+\frac{4}{3}\right) P_{\mathrm{eq}} \\
\frac{\mathrm{d}\left[\left(\mathrm{CH}_{3}\right)_{2} \mathrm{C}^{\cdot} \mathrm{OH}\right]}{\mathrm{d} t}= & -2 k_{\mathrm{t}}\left[\left(\mathrm{CH}_{3}\right)_{2} \mathrm{C}^{\cdot} \mathrm{OH}\right]^{2}+k_{\mathrm{q}}\left[{ }^{3} \mathrm{~K}\right]\left[\left(\mathrm{CH}_{3}\right)_{2} \mathrm{CHOH}\right] \\
\frac{\mathrm{d}\left[{ }^{3} \mathrm{~K}\right]}{\mathrm{d} t}= & -k_{\mathrm{a}}\left[{ }^{3} \mathrm{~K}\right]\left[\left(\mathrm{CH}_{3}\right)_{2} \mathrm{CHOH}\right]-k_{\mathrm{q}}\left[{ }^{3} \mathrm{~K}\right][\mathrm{Q}]
\end{aligned}
$$

Here $v$ represents the perpendicular magnetic moment in the rotating frame, $T_{1}$ and $T_{2}$ the radicals' relaxation times, $\omega_{1}$ the microwave field amplitude, $P_{\mathrm{G}}$ and $P_{\mathrm{F}}$ the geminate and F-pair RPM polarisation contributions in units of the radical equilibrium polarisation $P_{\mathrm{eq}}$. It is assumed that initially $v=0$ and $M_{z}=$ $=P_{\mathrm{G}} P_{\text {eq }}\left[\left(\mathrm{CH}_{3}\right)_{2} \mathrm{C}^{\circ} \mathrm{OH}\right]+M_{z}^{0}$. Solution of the equations gives the TREPR time profile on resonance of one line of the 2-hydroxy-2-propyl radical.

Reasonable values for many of the parameters may be obtained from the literature, which for the experimental conditions used are, $2 k_{t}=4 \cdot 10^{9} \mathrm{M}^{-1} \mathrm{~s}^{-1}$ [25], $T_{1} \approx T_{2} \approx 0.8 \mu \mathrm{s} \mathrm{[12],} \omega_{1}=4.6 \cdot 10^{5} \mathrm{rad} \cdot \mathrm{s}^{-1}$ [24], $P_{\mathrm{eq}}=250 \mathrm{~V} \cdot \mathrm{M}^{-1}$ [24] and $P_{\mathrm{F}}=3 P_{\mathrm{G}}=30$ for acetone $[12,26]$ and $P_{\mathrm{F}}=2 P_{\mathrm{G}}=30$ for benzophenone. The values of RPM polarisation used are for the $M_{I}= \pm 2$ lines and are smaller than given in the literature to take account of the lower viscosity used here (vide infra $\eta \approx 0.6 \mathrm{cP}$ ). As a simplification, for benzophenone it is assumed that the 2-hydroxy-2-propyl radicals only self terminate outside the geminate cage; because only the early time kinetics are of interest errors arising from this should be small. Typical calculated curves are similar to the experimental ones (vide infra). 


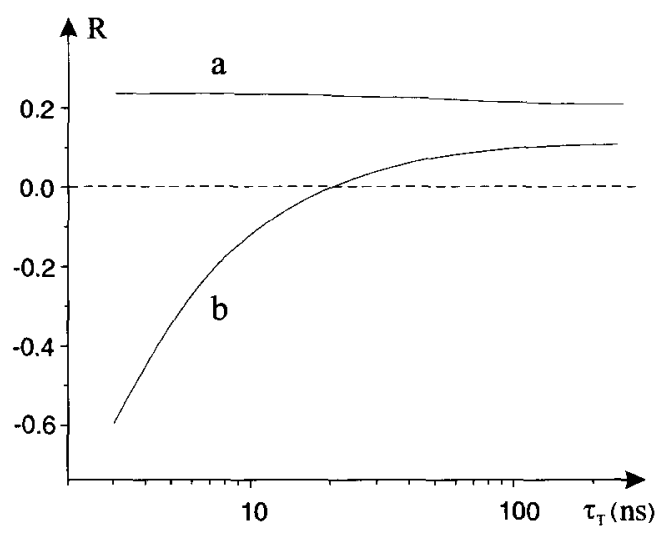

Fig. 1. Calculated net to multiplet polarisation ratio, $R$, versus triplet lifetime, $\tau_{\mathrm{T}}$, for the 2 -hydroxy2-propyl radical formed by photoreduction with (a) excited acetone and (b) excited benzophenone. The parameters used are given in the text.

To take account of the variation of radical concentration and therefore signal size with quencher concentration two time profiles, with opposite sign of RPM polarisation, were calculated for each condition. These are in effect simulations of the symmetric low and high field lines of the 2-hydroxy-2-propyl radical, and addition of the two profiles yields the net effect and subtraction the multiplet effect. It was found that the magnitude of the so-obtained multiplet effect at its maximum was to a good approximation proportional to the radical concentration for the concentration ranges used $\left(10^{-5}-10^{-4} \mathrm{M}\right)$, provided $P_{\mathrm{G}}$ and $P_{\mathrm{F}}$ were constant. It occurs because under these conditions at early time the multiplet polarisation arises predominately from geminate pairs and so its magnitude is proportional to the radical concentration. This useful result allows the size of the net signal, also measured at its maximum value, to be scaled to the radical concentration by division by the multiplet signal size. Calculations for different quencher concentrations gives a plot of the net/multiplet signal ratio with lifetime of the triplet showing the variation of the TM contribution. The lifetime of the triplet is given by

$$
\left.\tau_{\mathrm{T}}=\left(k_{\mathrm{a}}\left[{ }^{3} \mathrm{~K}\right]\left[\left(\mathrm{CH}_{3}\right)_{2} \mathrm{CHOH}\right]+k_{\mathrm{q}}{ }^{3} \mathrm{~K}\right][\mathrm{Q}]\right)^{-1} .
$$

The results of the calculation are shown in Fig. 1 for two situations: assuming the acetone triplet is not polarised by the TM; and assuming the benzophenone triplet has an initial polarization of $-60 \cdot P_{\text {eq }}$. In the acetone case the net/multiplet ratio is virtually constant with triplet lifetime as would be expected for a Boltzmann contribution. For long benzophenone $\tau_{\mathrm{T}}$ 's absorptive net polarisation is observed also arising from the Boltzmann population, however as $\tau_{\mathrm{T}}$ is reduced by quenching emissive polarisation is observed. If triplet acetone is assumed to be polarised in A, as observed for another aliphatic ketone [27], a similar curve to benzophenone is obtained but rising into greater $\mathrm{A}$ with decreasing 
$\tau_{\mathrm{T}}$. Thus quenching experiments should provide an easy test to see if radicals arising from a triplet precursor are polarised by the TM or not.

\section{Results}

Figure 2 shows the TREPR spectra recorded from benzophenone dissolved in benzene and $4 \mathrm{M}$ propan-2-ol with and without addition of pentadiene. Under these situations $\tau_{\mathrm{T}}$ was estimated as 250 and $5.5 \mathrm{~ns}$, respectively, using Eq. (12). In both cases the 2-hydroxy-2-propyl and benzylketyl radicals are observed in an E/A multiplet pattern as expected for triplet photoreduction. When $\tau_{\mathrm{T}}$ is reduced, Fig. $2 \mathrm{~b}$, the net polarisation changes from $\mathrm{A}$ to $\mathrm{E}$, mirroring the theoretical calculations and showing that for short $\tau_{\mathrm{T}}$ 's benzophenone exhibits emissive TM polarisation. Naturally the net E polarisation is also observed in the EPR time-profiles given in Fig. 3, which were acquired on-resonance on the low and high field lines of 2-hyroxy-2-propyl marked in Fig. 2. The emissive TM polarisation is in agreement with previous work [7].

Similar experiments were repeated for acetone and benzophenone with different quenchers and quencher concentrations to vary $\tau_{\mathrm{T}}$. In all cases the quencher concentration was small and, therefore, unlikely to alter the solution composition and the RPM polarisation magnitudes. This allows a plot analogous plot to Fig. 1 to be made. For evaluation, the maximum signal amplitudes of the low and high field lines were determined from the time profiles, then the sum of the amplitudes was divided by the difference and plotted versus $\tau_{\mathrm{T}}$, as calculated from

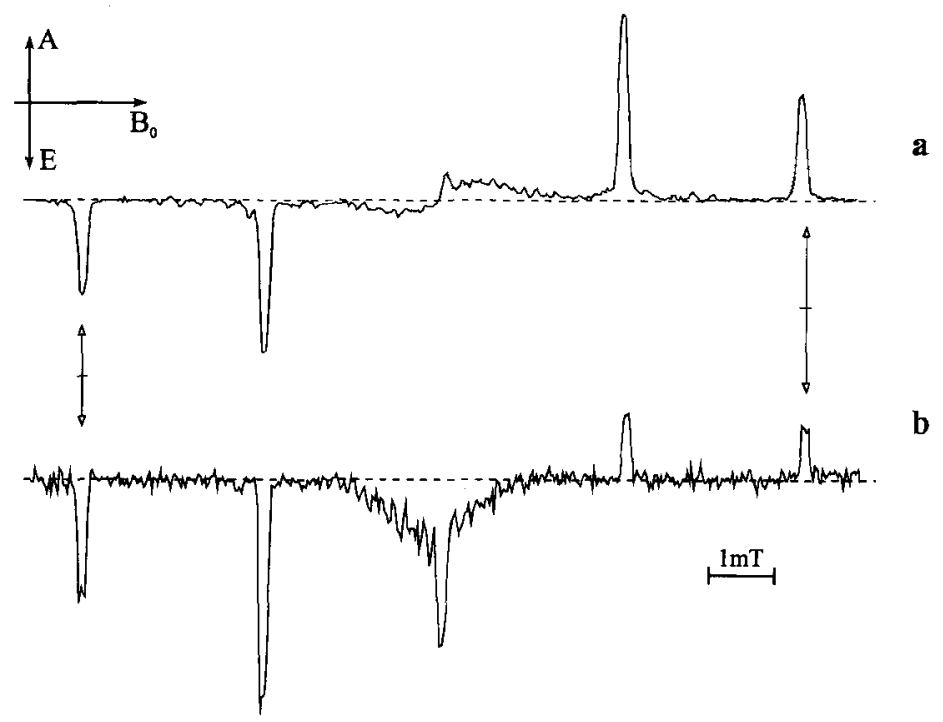

Fig. 2. TREPR recorded $0.4-0.6 \mu$ s post flash from solution of a $58.6 \mathrm{mM}$ benzophenone and $4 \mathrm{M}$ propan-2-ol in benzene and $b$ the same solution with trans-1,3-pentadiene $36.6 \mathrm{mM}$. 

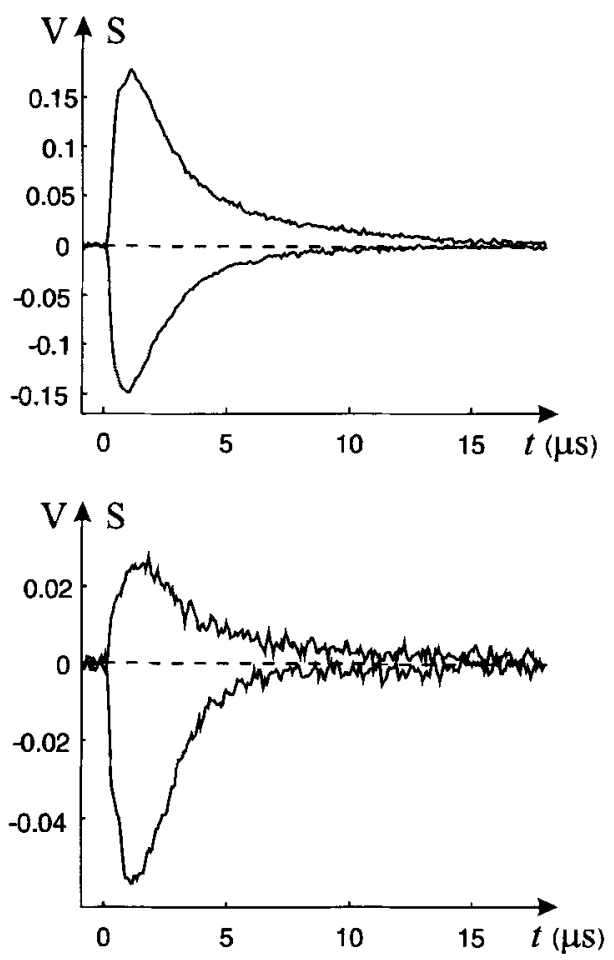

Fig. 3. TREPR time profiles signal $S$ in $V$ versus time in $\mu$ s, recorded on resonance for the high and low field lines marked in Fig. 2. The experimental conditions for $\mathbf{a}$ and $\mathbf{b}$ are given in Fig. 2.

Eq. (12). For acetone an additional term was included in Eq. (12) to take account of triplet quenching by benzene [20]. The results are shown in Fig. 4 and immediately demonstrate that the 2-hydroxy-2-propyl radicals arising from acetone photoreduction are not polarised by the TM, because the net/multiplet ratio is invariant with $\tau_{\mathrm{T}}$ in the experimentally accessible range of 6-250 ns. By comparison with benzophenone, if a TM contribution where present it should be observable in this range. Thus, acetone either has a much shorter ${ }^{3} T_{1}$ than benzophenone, perhaps due to its smaller size [6], or little TM polarisation is initially produced in the acetone triplet.

The experimental results show a good quantitative match to the theoretical curves of Fig. 1, which are redisplayed in Fig. 4. This indicates that the parameters and assumptions used are reasonable. In particular the conclusion that the radical concentration is proportional to the size of the multiplet effect is verified. It must be emphasized that this is only the case when the quencher is present in small amounts and the solvent composition is not altered. Attempts to repeat the experiments by varying the propan-2-ol concentration failed for this reason. No attempts were made to fit the experimental time profiles as this requires a knowledge of the absolute radical concentrations which were unknown. 


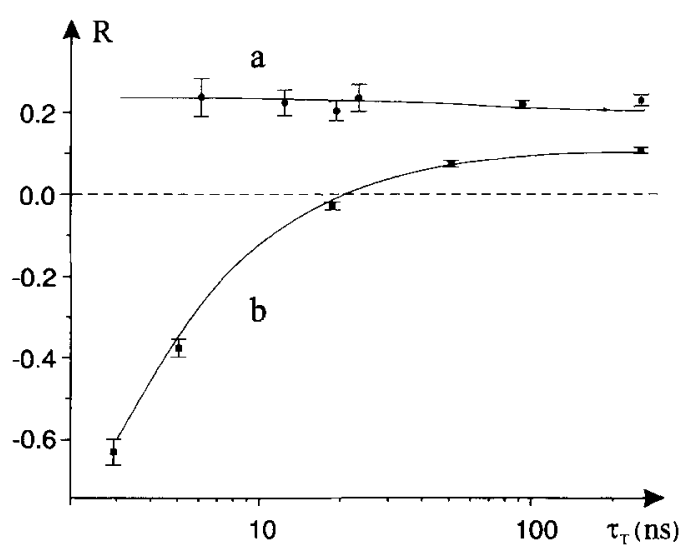

Fig. 4. Experimental net to multiplet polarisation ratio, $R$, versus triplet lifetime, $\tau_{\mathrm{T}}$, for the 2-hydroxy-2-propyl radical formed by photoreduction with (a) excited acetone and (b) excited benzophenone. In (a) the quenchers used were $31 \mathrm{mM}$ trans-1,3-pentadiene, $8 \mathrm{mM}$ fumaronitirile, $11 \mathrm{mM}$ azon-butane, $50 \mathrm{mM}$ azo-tert-butane, no quencher, and no quencher for the points from left to right. Solution contained $1.6 \mathrm{M}$ acetone and $4 \mathrm{M}$ propan-2-ol in benzene, except for the point at $\tau_{\mathrm{T}}=250$ ns where cyclohexane was the solvent. In (b) the quenchers were 63, 36.6, $9.2 \mathrm{mM}$ trans-1,3pentadiene, $20.3 \mathrm{mM}$ azo-tert-butane, and no quencher for the points from left to right. Solutions contained $58.6 \mathrm{mM}$ benzophenone and $4 \mathrm{M}$ propan-2-ol in benzene.

The acetone result is in agreement with previous quantitative attempts to measure the size of the net polarisation [9, 12] and an investigation of triplet acetone polarisation by fast reaction with triethylamine [28]. However it is in disagreement with some qualitative experiments which suggested the TM was operative [3, 14]. In particular it has been argued that TREPR spectrometers are too insensitive to detect Boltzmann signals, the current results show this view is too pessimistic. The discrepancy in conclusions may have arisen because of the use of different laser powers, focusing and optical densities by different groups. The acetone photosystem is known to be sensitive to extremely fast 2-photon $\alpha$-cleavage at high laser powers [27, 29], which could produce radicals within ${ }^{3} T_{1}$ giving TM polarisation. Additionally high laser powers produce high concentrations of triplets leading to substantial triplet-triplet annihilation. This reaction can lead to radical formation and because it is spin selective could produce some net polarisation. These two side reactions lead to production of radicals with different $g$-factors to 2-hydroxy-2-propyl and then net $\Delta g$ RPM polarisation must also be considered. Nevertheless the current work shows that at low laser powers and optical densities no TM is observed in the photoreduction of acetone with propan-2-ol.

Variation of the quencher between trans-1,3-pentadiene, fumaronitrile, azo-n-butane and azo-tert-butane did not alter the results, adding further support to the conclusions. These quenchers do not produce radicals and appear to be reliable choices for such experiments. AIBN was also investigated as a possible choice, which on quenching produces 2-cyano-2-propyl radicals according to reaction 


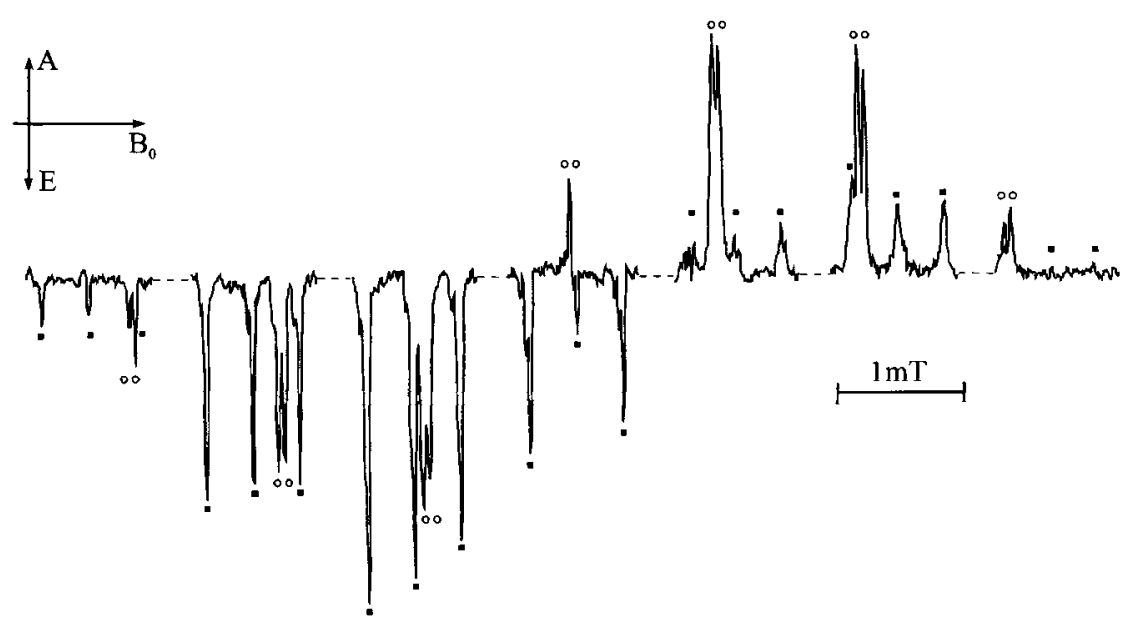

Fig. 5. TREPR spectrum recorded $1.3-1.9 \mu$ s post flash from a solution of $1.6 \mathrm{M}$ acetone, $4 \mathrm{M}$ propan-2-ol and $20 \mathrm{mM}$ AIBN in benzene. Lines marked with a circle and square arise from 2-cyano-2-propyl and 2-hydroxy-2-propyl radicals, respectively.

Eq. (1). Here, any net polarisation should be passed to the 2-cyano-2-propyl radicals provided the reactions are not spin selective. Figure 5 shows the spectra acquired with acetone/propan-2-ol, where both the 2-hydroxy-2-propyl and 2cyano-2-propyl are observed in an E/A RPM multiplet pattern. However, whilst the 2-hydroxy-2-propyl radicals show a small net A polarisation, the 2-cyano-2propyl radicals clearly show a much larger net $\mathrm{E}$ contribution. This polarisation cannot be due to net $\Delta g$ RPM polarisation produced in a 2-cyano-2-propyl/2hydroxy-2-propyl radical pair as the $g$-factor of the radicals, 2.0031 and 2.0028 respectively [30], would produce net A polarisation in 2-cyano-2-propyl. Neither can the net signal be due to TM in acetone as there is none. Thus one of the reactions in 2-cyano-2-propyl production must be spin selective producing polarisation. This may be either the quenching itself or a process in the decomposition of the azo-compound.

Years ago, Steiner $[31,32]$ observed a magnetic field effect on the radical ion yield in electron-transfer reactions between dye triplets and heavy-atom substituted electron donors, forming triplet exciplexes with each other. The effect was explained in terms of a reversed TM, i.e., a preferred deactivation of certain zerofield states of the triplet exciplex via intersystem crossing to the ground state, thus producing spin polarisation in the molecular frame of the exciplex. A nonvanishing zero-field splitting should partially transform this polarisation into the laboratory frame and yield an initial net electron spin polarisation of the radicals, formed in the competing dissociation of the exciplex. In fact, as predicted by Steiner [33], this spin polarisation seems to have been experimentally observed recently [34-36], although the authors renamed the mechanism spin-orbit coupling induced electron spin polarisation. 
The initial net emission observed here for the 2-cyano-2-propyl radicals might also well be due to such a reversed TM in triplet AIBN. The kinetic conditions for the effect to occur are nearly ideal for AIBN, as triplet sensitisation leads to fast cleavage, but with quantum yields of only $0.1-0.15$, leaving intersystem crossing to the ground state as the faster dominating route of deactivation [15]. Also the magnitude of the emission, which is estimated to about $-15 \cdot P_{\text {eq, }}$, would be compatible with such a polarisation mechanism.

Of course, further data is required to corroborate the assignment of the unusual net emission after triplet sensitisation of AIBN to a reversed TM. At least our results show that AIBN is not a suitable quencher for checking polarisations of other triplet molecules because it produces polarisation itself. A previous measurement of triplet spin relaxation [4], using quenching with AIBN, must therefore be re-examined in light of this.

\section{Conclusions}

Quenching experiments provide an easily applied method to determine if a radical observed by TREPR shows TM polarisation, provided a benign triplet quencher is chosen. Using this technique it is found that the acetone/propan-2-ol system does not show TM polarisation and the net A contribution is due to the Boltzmann population of the triplet or radical. AIBN is not a suitable compound for such experiments as it produces polarisation, probably via a reversed TM.

\section{Acknowledgements}

This investigation was undertaken in the frame of an INTAS project (93-1626ext) on magnetic spin effects in chemical reactions. The authors thank Prof. U. E. Steiner (Konstanz) and Dr. A. I. Sushin (Moscow) for useful discussions and gratefully acknowledge financial support by the Swiss National Foundation for Scientific Research.

\section{References}

[1] Turro N.J.: Modern Molecular Photochemistry. Mill Valley, CA: University Science Books 1991.

[2] McLauchlan K.A., Yeung M.T.: Roy. Soc. Chem. Spec. Rep. Electron Spin Reson. 14, 32-64 (1994)

[3] Shkrob I.A., Wan J.K.S.: Res. Chem. Intermed. 17, 77-100 (1992)

[4] Goudsmit G.-H., Paul H.: Chem. Phys. Lett. 260, 453-457 (1996)

[5] Atkins P.W., Dobbs A.J., McLauchlan K.A.: Chem. Phys. Lett. 29, 616-617 (1974)

[6] Atkins P.W., Evans G.T.: Mol. Phys. 27, 1633-1644 (1974)

[7] Akiyama K., Sekiguchi S., Tero-Kubota S.: J. Phys. Chem. 100, 180-183 (1996)

[8] Beckert D., Schneider G.: Chem. Phys. 116, $421-428$ (1987)

[9] van Willigen H., Levstein P.R., Ebersole M.H.: Chem. Rev. 93, 173-197 (1993)

[10] MeLauchlan K.A., Simpson N.J.K., Smith P.D.: Res. Chem. Intermed. 16, $26-46$ (1991)

[11] Hirota N., Tominaga K., Yamauchi S.: Bull. Chem. Soc. Jpn. 68, 2997-3010 (1995) 
[12] Paul H.: Chem. Phys. 40, 265-274 (1979); ibid. 43, 294 (1979)

[13] Yamauchi, S., Tominaga K., Hirota N.: J. Phys. Chem. 90, 2367-2369 (1986)

[14] Batchelor S.N., Kay C.W.M., McLauchlan K.A., Smith P.D., Yeung M.T.: Mol. Phys. 82, 325342 (1994)

[15] Engel P.S.: Chem. Rev. 80, 99-150 (1980)

[16] Takemura T., Ohara K., Murai H., Kuwata K.: Chem. Lett. 1990, 1635-1638.

[17] Jent F., Paul H.: Chem. Phys. Lett. 160, 632-639 (1989)

[18] Murov S.L.: Handbook of Photochemistry. New York: Marcel Dekker 1973.

[19] Scaiano J.C.: Handbook of Organic Photochemistry. Boca Raton: CRC Press 1989.

[20] Wamser C.C., Medary R.T., Kochevar I.E., Turro N.J., Chang P.L.: J. Am. Chem. Soc. 97, 4864 4869 (1975)

[21] Turro N.J., Tanimoto Y.: J. Photochem. 14, 199-203 (1980)

[22] Pedersen J.B.: J. Chem. Phys. 59, 2656-2667 (1973)

[23] Verma N.C., Fessenden R.W.: J. Phys. Chem. 65, 2139-2155 (1976)

[24] Savitsky A.N., Paul H.: Appl. Magn. Reson. 12, 449 (1997)

[25] Lehni M., Fischer H.: Int. J. Chem. Kinet. 15, 733-757 (1983)

[26] Levstein P.R., van Willigen H.: J. Chem. Phys. 95, 900-907 (1991)

[27] Batchelor S.N., Fischer H.: J. Chem. Phys. 100, 556-564 (1996)

[28] Ohara K., Hirota N., Steren C.A., van Willigen H.: Chem. Phys. Lett. 232, 169-175 (1995)

[29] Jent J., Paul H., Fischer H.: Chem. Phys. Lett. 146, 315-319 (1988)

[30] Landolt-Börnstein: Magnetic Properties of Free Radicals (Fischer H., Hellwege K.H., eds.). New Series, volumes II/1, II/9b, II/17b. Berlin: Springer 1965-1986.

[31] Steiner U.: Chem. Phys. Lett. 74, 108-111 (1980)

[32] Steiner U.E., Ulrich T.: Chem. Rev. 89, 51-147 (1989)

[33] Steiner U.E.: Ber. Bunsenges. Phys. Chem. 85, 228-233 (1981)

[34] Katsuki A., Akiyama K., Ikegami Y., Tero-Kubota S.: J. Am. Chem. Soc. 116, 12065-12066 (1994)

[35] Katsuki A., Akiyama K., Tero-Kubota S.: Bull. Chem. Soc. Jpn. 65, 3383-3389 (1995)

[36] Sasaki S., Katsuki A., Akiyama K., Tero-Kubota S.: J. Am. Chem. Soc. 119, 1323-1327 (1997)

Author's address: Prof. Dr. Henning Paul, Physikalisch-Chemisches Institut der Universität Zürich, Winterthurerstrasse 190, CH-8057 Zürich, Switzerland 